\title{
Epidemiological, clinical and laboratory differences between male urethral infections due to Haemophilus spp. and those due to Neisseria gonorrhoeae, Chlamydia trachomatis, Mycoplasma genitalium and Ureaplasma urealyticum: A descriptive study
}

\author{
Alvaro Vives, Marco Cosentino, Lluis Bassas, Carles Alonso, Felix Millan \\ Sexually Transmitted Infection Department, Andrology Service, Fundació Puigvert, Universitat Autònoma de Barcelona, \\ Barcelona, Spain.
}

\begin{abstract}
Summary Objective: To describe the epidemiological, clinical and laboratory characteristics of male patients diagnosed with Haemophilus spp. urethral infection and to compare them with the characteristics of male patients diagnosed with N. gonorrhoeae, C. trachomatis, M. genitalium and U. urealyticum urethral infection. Over the past 2 years, an increase in urethral infections due to Haemophilus spp. was observed.

Materials and methods: All male patients who attended our Department of Sexually Transmitted Infections between January 2018 and February 2019 were retrospectively studied; they underwent conventional bacteriological and multiplex PCR studies in the urethra at the same time.

Results: Of the 86 patients studied, a unique microorganism was detected in 76 cases, $N$. gonorrhoeae in 24, Haemophilus spp. in 21 (16 H. parainfluenzae and $5 \mathrm{H}$. influenzae), C. trachomatis in 19, M. genitalium in 8 and $U$. urealyticum in $4 ; 10$ cases presented more than one microorganism. In case of multiple aetiological agents, sexual partnership was multiple. In the Haemophilus group, $81 \%$ reported only unprotected oral insertive sex; symptoms lasted for more than one week in $62 \%$ of the patients.

Conclusions: Haemophilus is an aetiological agent of non-gonococcal urethritis whose incidence is clearly increasing; the main route of transmission is oral sex. The most common reason for consultation is dysuria and testicular pain, while urethral discharge was predominant for the other causes of urethral infection. Due to the high frequency of antibiotic resistance in the Haemophilus group, it is necessary to confirm eradication by performing a test of cure.
\end{abstract}

KEY WORDS: Haemophilus spp.; Neisseria gonorrhoeae; Chlamydia trachomatis; Mycoplasma genitalium; Ureaplasma urealyticum; Urethral infections; Males; Sexually transmitted infections.

Submitted 15 August 2021; Accepted 7 October 2021

\section{INTRODUCTION}

One of the most common presentations of sexually transmitted infections (STI) among men is acute urethritis. Classically, cases of sexually transmitted urethritis have been classified according to their aetiology, as either gono- coccal urethritis (GU), caused by Neisseria gonorrhoeae, or non-gonococcal urethritis (NGU), caused by other aetiological agents, such as Chlamydia trachomatis, Mycoplasma genitalium or Ureaplasma urealyticum (1). However, it is estimated that the aetiology remains unknown in up to $30 \%-40 \%$ of cases of NGU (2).

In recent years, new microorganisms have been described as aetiological agents for NGU, with bacteria of the genus Haemophilus taking on special importance $(3,4)$. Sexual transmission to the urethra via insertive oral sex is recognized as a potential mode of transmission (5). However, this mechanism has not been fully proven.

We conducted a retrospective study to describe the epidemiological, clinical and laboratory characteristics of male patients diagnosed with urethral infections due to Haemophilus spp. and to compare these characteristics with those observed in male patients diagnosed with urethral infections due to N. gonorrhoeae, C. trachomatis, M. genitalium and U. urealyticum.

\section{MAterials AND Methods}

\section{Inclusion criteria}

The study population comprised all male patients who attended our Department of Sexually Transmitted Infections (STI) between January 2018 and February 2019 and underwent conventional bacteriological and multiplex PCR studies in the urethra at the same time. The study was approved by our institutional medical and research ethics committee.

Demographic, behavioural, clinical and laboratory data were obtained by reviewing medical charts. The different parameters under study were defined as follows:

- Sexual orientation.

- Type of sexual partner.

- Number of sexual partners during the 90 days prior to the infection.

- Type of unprotected sex during the 90 days prior to the infection.

- History of STIs: all patients were asked about their STI history. 
- HIV status.

- Recreational drugs during the past year.

- Main reason for consultation.

- Duration of symptoms.

- Presence of leukocytes.

- Types of treatment

- Test of cure: This consists in the performance of a conventional bacteriological and/or multiplex PCR study 3 weeks after the end of the treatment.

- Clinical cure: in our STI Department patient is seen again 3 weeks after the end of the treatment. All patients without signs and symptoms at that time are considered clinically cured.

- Partner notification and treatment: We considered a positive result for partner notification when at least one of the sexual partners from the last 90 days had been advised and treated correctly.

According to the CDC STI guidelines, urethritis can be documented on the basis of any of the following signs or laboratory tests:

1) Mucoid, mucopurulent, or purulent discharge on examination

2) Gram stain of urethral secretions exist that demonstrate $\geq 2$ WBCs per oil immersion field

3) Positive leukocyte esterase test on first-void urine or microscopic examination of sediment from a spun first-void urine demonstrating $\geq 10 \mathrm{WBCs} / \mathrm{HPF}$.

Men evaluated in settings in which Gram stain or MB or GV smear is unavailable who meet at least one criterion for urethritis (i.e., urethral discharge, positive leukocyte esterase test on first void urine, or microscopic examination of first-void urine sediment with $\geq 10 \mathrm{WBCs} / \mathrm{HPF}$ ) should be tested for C. trachomatis and N. gonorrhoeae by NAATs.

\section{Laboratory studies}

Only urethral samples were obtained: the first sample for culture and leukocyte counting while the second for multiple PCR. Conventional bacteriological study included direct examination by Gram stain of the sample smear under 100x and 1000x magnification, and culture. Petri dishes of chocolate agar (BD), Martin-Lewis agar (BD), Gardnerella agar (BD) and Sabouraud with gentamicin and chloramphenicol (BD) were used. Chocolate agar, Martin-Lewis agar and Gardnerella agar were incubated at $35^{\circ} \mathrm{C}$ with enriched $7 \% \mathrm{CO}_{2}$ atmosphere for 3 days (chocolate and Martin-Lewis agar) or 2 days (Gardnerella agar). Sabouraud was incubated at $35^{\circ} \mathrm{C}$ for 2 days at room air. N. gonorrhoeae and Haemophilus strains were identified by API NH test (bioMérieux). Multiplex PCR was performed by AnyplexTM II STI-7 assay (Seegene), which detects five microorganisms in a single reaction: Chlamydia trachomatis, Neisseria gonorrhoeae, Trichomonas vaginalis, Mycoplasma genitalium, Ureaplasma urealyticum. Leukocytes and other elements were looked for in LPF microscopy (100x). Then, leukocytes in 20 fields of these areas were observed at $1000 \mathrm{x}$ and the average was calculated. The antimicrobial susceptibility of N. gonorrhoeae and Haemophilus spp. strains was analyzed according to 2015 CLSI guidelines (6).

\section{Statistical analysis}

Statistical analysis was conducted using Statistical Product and Service Solutions Version 18.0 (SPSS). Descriptive statistics were used to evaluate the study outcomes. Mean values and ranges are presented for continuous measurements. Frequencies and percentages were reported for dichotomous and ordinal variables. Differences of discrete variables were analysed with unpaired two-tailed Student's t test. Comparisons of proportions were performed with Fisher's exact test. Statistical significance was set at $\mathrm{p}<0.05$. Multivariate binary logistic regressions were used to search for the better combination of variables to predict the aetiology of Haemophilus spp, and the accuracy of the model was measured as the area under the ROC curve (AUC).

\section{Results}

In total, 158 patients met the inclusion criteria, all had symptoms except for 5 who attended for screening. The screening cases were men to whom a sexual partner of the last 90 days had warned a positive result for: N. gonorrhoeae, C. trachomatis, M. genitalium, U. urealyticum.

We obtained 86 (54\%) positive results for the microor-

Table 1.

Reasons for consultation.

\begin{tabular}{|l|c|c|}
\hline Reason for consultation & Total & Positive cases ${ }^{*}$ \\
\hline Urethral secretion & 69 & $55(86.2 \%)$ \\
\hline Dysuria & 47 & $20(42.5 \%)$ \\
\hline Testicular pain & 9 & $6(66.7 \%)$ \\
\hline Balanitis & 12 & $0(0 \%)$ \\
\hline Meatitis & 9 & $2(22.2 \%)$ \\
\hline Screening & 5 & $2(40 \%)$ \\
\hline Hematospermia & 6 & $1(16.7 \%)$ \\
\hline Urethrorrhagia & 1 & $0(0 \%)$ \\
\hline Total & 158 & $86(54.4 \%)$ \\
\hline $\begin{array}{l}\text { "Positive results for any of the following microorganisms: Haemophilus, Neisseria gonorrhoeae, } \\
\text { Chlamydia trachomatis, Mycoplasma genitalium or Ureaplasma urealyticum. }\end{array}$ \\
\hline
\end{tabular}

Table 2.

Classification of the isolated microorganisms.

\begin{tabular}{|c|c|c|}
\hline \multicolumn{2}{|l|}{ Microorganism } & \multirow{3}{*}{$\begin{array}{c}\text { Number } \\
21\end{array}$} \\
\hline \multirow[t]{2}{*}{ Haemophilus spp. } & Haemophilus parainfluenzae 16 & \\
\hline & Haemophilus influenzae & \\
\hline \multicolumn{2}{|c|}{ Neisseria gonorrhoeae } & 24 \\
\hline \multicolumn{2}{|c|}{ Chlamydia trachomatis } & 19 \\
\hline \multicolumn{2}{|c|}{ Mycoplasma genitalium } & 8 \\
\hline \multicolumn{2}{|c|}{ Ureaplasma urealyticum } & 4 \\
\hline \multicolumn{2}{|c|}{ C. trachomatis + M. genitalium } & 1 \\
\hline \multicolumn{2}{|c|}{ C. trachomatis + N. gonorrhoeae } & 1 \\
\hline \multicolumn{2}{|c|}{ C. trachomatis + H. parainfluenzae } & 3 \\
\hline \multicolumn{2}{|c|}{ C. trachomatis + H. influenzae } & 2 \\
\hline \multicolumn{2}{|c|}{ U. urealyticum + H. parainfluenzae } & 1 \\
\hline \multicolumn{2}{|c|}{ U. urealyticum + Gardnerella vaginalis } & 1 \\
\hline \multicolumn{2}{|c|}{ N. gonorrhoeae + M. genitalium + U. urealyticum } & 1 \\
\hline
\end{tabular}


ganisms under study however, when we focused the main reason for consultation. In patients with urethral discharge the percentage of positives increased to $86.2 \%$ (55 cases) (Table 1).

Regarding the 86 positive results, a single microorganism was detected in 76 cases; 24 cases of $N$. gonorrhoeae, 21 cases of Haemophilus spp. (16 H. parainfluenzae and $5 \mathrm{H}$. influenzae), 19 cases of C. trachomatis, 8 cases of M. genitalium and 4 cases of $U$. urealyticum.

Ten cases presented with more than one microorganism, being C. trachomatis the most frequent, followed by Haemophilus spp. (Table 2). In Table 3 we described the demographic, behavioural, clinical and laboratory data according to the germ obtained or multiple infection.

\section{Demographic and behavioural differences}

Age: The mean age of the patients included in the study was 31.6 years (range 16-74). The lowest mean age (24.5 years) was observed in patients with infection by $U$. urealyticum while the highest was seen in those with $N$. gonorrhoeae infection (37.5 years); Haemophilus cases displayed the second highest mean age.

Sexual orientation: For all microorganisms, including multiple infections, most cases occurred in males having sex

Table 3.

Reasons for consultation.

\begin{tabular}{|c|c|c|c|c|c|c|c|c|}
\hline \multicolumn{2}{|l|}{ Aetiology } & $\begin{array}{l}\text { Haemophilus } \\
\text { spp }\end{array}$ & $\begin{array}{l}\text { Neisseria } \\
\text { gonorrhoeae }\end{array}$ & $\begin{array}{l}\text { Chlamydia } \\
\text { trachomatis }\end{array}$ & $\begin{array}{l}\text { Mycoplasma } \\
\text { genitalium }\end{array}$ & $\begin{array}{l}\text { Ureaplasma } \\
\text { urealyticum }\end{array}$ & $\begin{array}{l}\text { Multiple } \\
\text { infection }\end{array}$ & $\mathbf{P}^{*}$ \\
\hline \multicolumn{2}{|l|}{ Number of cases } & 21 & 24 & 19 & 8 & 4 & 10 & - \\
\hline \multicolumn{2}{|l|}{ Mean age (years) } & $35.6(20-52)$ & $37.5(21-74)$ & $29.8(16-57)$ & $32.6(21-47)$ & $24.5(16-38)$ & $30.1(18-46)$ & $p=0.315$ \\
\hline \multirow{3}{*}{ Sexual orientation } & MSW & $13(62 \%)$ & $14(58 \%)$ & $16(84 \%)$ & $7(88 \%)$ & $4(100 \%)$ & $7(70 \%)$ & $p=0.459$ \\
\hline & MSM & $7(33 \%)$ & $10(42 \%)$ & $3(16 \%)$ & - & - & $1(10 \%)$ & $p=0.616$ \\
\hline & BSM & $1(5 \%)$ & - & - & $1(12 \%)$ & - & $2(20 \%)$ & $p=0.560$ \\
\hline \multirow[t]{2}{*}{ Type of sexual partner } & Single & $5(24 \%)$ & $8(33 \%)$ & $9(47 \%)$ & $4(50 \%)$ & - & $2(20 \%)$ & $p=0.381$ \\
\hline & Multiple & $16(76 \%)$ & $16(67 \%)$ & $10(53 \%)$ & $4(50 \%)$ & $4(100 \%)$ & $8(80 \%)$ & $p=0.381$ \\
\hline \multicolumn{2}{|l|}{ Number of sexual partners } & $3.5(1-20)$ & $2.5(1-10)$ & $2.4(1-10)$ & $3 ., 6(1-20)$ & $2.2(1-4)$ & $8.4(1-20)$ & $p=0.336$ \\
\hline \multirow{3}{*}{$\begin{array}{l}\text { Type of unprotected sex durings } \\
\text { the past } 90 \text { day }\end{array}$} & UOIS & $21(100 \%)$ & $24(100 \%)$ & $18(95 \%)$ & $8(100 \%)$ & $4(100 \%)$ & $10(100 \%)$ & $p=0.676$ \\
\hline & UVS & $4(19 \%)$ & $9(38 \%)$ & $14(74 \%)$ & $7(88 \%)$ & $3(75 \%)$ & $7(70 \%)$ & $p=0.004$ \\
\hline & UAIS & $1(5 \%)$ & $4(17 \%)$ & - & $1(12 \%)$ & - & $1(10 \%)$ & $p=0.922$ \\
\hline \multicolumn{2}{|l|}{ Previous history of STI } & $9(43 \%)$ & $7(29 \%)$ & $8(42 \%)$ & $2(22 \%)$ & - & $5(50 \%)$ & $p=0.477$ \\
\hline \multirow[t]{3}{*}{ HIV status } & Positive & $2(10 \%)$ & - & $2(11 \%)$ & - & - & $1(10 \%)$ & $p=0.651$ \\
\hline & Negative & $14(66 \%)$ & $21(87 \%)$ & $16(84 \%)$ & $8(100 \%)$ & $3(75 \%)$ & $9(90 \%)$ & $p=0.081$ \\
\hline & Not available & $5(24 \%)$ & $3(13 \%)$ & $1(5 \%)$ & - & $1(25 \%)$ & - & $p=0.182$ \\
\hline \multirow[t]{3}{*}{ Recreational drugss } & Alcohol & $18(86 \%)$ & $22(92 \%)$ & $18(95 \%)$ & $8(100 \%)$ & $4(100 \%)$ & $8(80 \%)$ & $p=0.398$ \\
\hline & Tobacco & $14(66 \%)$ & $11(46 \%)$ & $12(63 \%)$ & $5(63 \%)$ & $2(50 \%)$ & $6(60 \%)$ & $p=0.546$ \\
\hline & Other drugs & $3(14 \%)$ & $3(13 \%)$ & $3(16 \%)$ & - & $1(25 \%)$ & $4(40 \%)$ & $p=0.794$ \\
\hline \multirow[t]{6}{*}{ Main reason for consultation } & Discharge & $7(33 \%)$ & $23(96 \%)$ & $13(68 \%)$ & $4(50 \%)$ & $1(25 \%)$ & $8(80 \%)$ & $p=0.003$ \\
\hline & Dysuria & $8(38 \%)$ & $1(4 \%)$ & $4(21 \%)$ & $3(38 \%)$ & $2(50 \%)$ & $1(10 \%)$ & $p=0.132$ \\
\hline & Testicular pain & $4(19 \%)$ & - & $2(11 \%)$ & - & - & - & $p=0.096$ \\
\hline & Meatitis & $1(5 \%)$ & - & - & - & - & $1(10 \%)$ & \\
\hline & Haematospermia & $1(5 \%)$ & - & - & - & - & - & \\
\hline & Screening & - & - & - & $1(12 \%)$ & $1(25 \%)$ & - & \\
\hline \multirow[t]{3}{*}{ Duration of symptoms } & $<1$ week & $8(38 \%)$ & $22(92 \%)$ & $11(58 \%)$ & $6(75 \%)$ & $2(50 \%)$ & $8(80 \%)$ & $p=0.006$ \\
\hline & $>1$ week & $13(62 \%)$ & $2(8 \%)$ & $8(42 \%)$ & $1(12 \%)$ & $1(25 \%)$ & $2(20 \%)$ & $p=0.002$ \\
\hline & Not available & - & - & - & $1(12 \%)$ & $1(25 \%)$ & - & \\
\hline \multicolumn{2}{|l|}{ Presence of leukocytes } & $5(24 \%)$ & $18(75 \%)$ & $8(42 \%)$ & - & - & $4(40 \%)$ & $p=0.124$ \\
\hline \multirow[t]{8}{*}{ Treatments } & Ceftriaxone (Ceft.) & - & 4 & - & - & - & - & - \\
\hline & Ceft. + doxycycline & 2 & 16 & 8 & - & 2 & 4 & - \\
\hline & Ceft. + azithromycin & 1 & 4 & 2 & - & - & 1 & . \\
\hline & Doxycycline & 2 & - & 4 & - & 2 & 4 & . \\
\hline & Azithromycin, single $1 \mathrm{~g}$ oral dose & - & - & 5 & - & - & - & - \\
\hline & Azithromycin 5-day course & 3 & - & - & 4 & - & 1 & - \\
\hline & Moxifloxacin & - & - & - & 4 & - & - & . \\
\hline & Other & 13 & - & - & - & - & - & . \\
\hline \multirow[t]{2}{*}{ Clinical cure } & Yes & $19(90 \%)$ & $23(96 \%)$ & $17(89 \%)$ & $8(100 \%)$ & $3(75 \%)$ & $10(100 \%)$ & $p=0.968$ \\
\hline & Not known & $2(10 \%)$ & $1(4 \%)$ & $2(11 \%)$ & - & $1(25 \%)$ & - & $p=0.968$ \\
\hline \multicolumn{2}{|l|}{$\overline{\text { Culture/PCR control }}$} & $19(90 \%)$ & - & - & $8(100 \%)$ & - & $10(100 \%)$ & $\cdot$ \\
\hline \multicolumn{2}{|l|}{ Partner notification and treatment } & - & $23(96 \%)$ & $18(95 \%)$ & $8(100 \%)$ & $3(75 \%)$ & $10(100 \%)$ & - \\
\hline \multicolumn{9}{|c|}{ 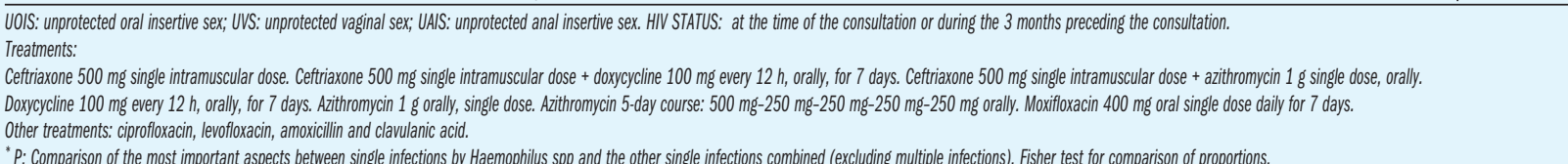 } \\
\hline
\end{tabular}


with women (MSW). Of the cases of Haemophilus spp. and N. gonorrhoeae, 33\% and 42\% respectively occurred in males having sex with males (MSM). No cases of infection by U. urealyticum or M. genitalium were found in MSM. Type of sexual partner: For most aetiological agents, the type of sexual partner was multiple; an exception was $M$. genitalium infection, for which $50 \%$ of patients reported a single partner and 50\% multiple partners.

Number of sexual partners: The highest mean number of sexual partners in the last 90 days was observed in the multiple infection group (8.4), followed by M. genitalium (3.6) and Haemophilus spp. (3.5), with a range from 1 to 20. Type of unprotected sex: between 95 and $100 \%$ of all patients had unprotected oral insertive sex, being the most frequent risk sexual practice, without differences between the different germs. On the other hand, only $19 \%$ of the Haemophilus spp reported unprotected vaginal sex, which is statistically significant in relation to the other germs $(\mathrm{p}=0.004)$ (Table 3$)$.

History of STIs: Between 22\% and 50\% of the patients had a history of STIs, without significant differences according to the microorganism, with the exception of patients with $U$. urealyticum infection, who had no history of STIs. HIV status: In 76 of the 86 cases (88\%) the HIV status was known; only 5 patients were HIV+, and all of them were under treatment with negative viral load.

Recreational drugs: Between $80 \%$ and $100 \%$ of the patients consumed alcohol on a regular basis and between $46 \%$ and $66 \%$ used tobacco, without significant differences between the various groups. Use of other drugs ranged between $13 \%$ and $40 \%$, being higher in patients with multiple infections.

\section{Clinical differences}

Main reason for consultation: Of the 158 cases analysed, all were symptomatic except for 5 who consulted for screening. Among patients with single infections other than Haemophilus spp., urethral discharge was the main reason for consultation with a statistically significant difference observed in relation to the Haemophilus spp. group ( $\mathrm{p}=$ 0.003 ); dysuria was the main reason for consultation in $38 \%$, urethral discharge in 33\%, testicular pain in $19 \%$,

\section{Table 4.}

Antibiotic resistance in patients with $\mathrm{H}$. parainfluenzae and $\mathrm{H}$. influenzae infections.

\begin{tabular}{|c|c|c|c|c|c|c|}
\hline Antibiotic & $\begin{array}{r}\text { H. } \\
\text { Cases }\end{array}$ & $\begin{array}{l}\text { Jarainfluenz } \\
\text { Sensitive }\end{array}$ & $\begin{array}{l}z \text { zae } \\
\text { Resistant }\end{array}$ & $\begin{array}{r}\text { H } \\
\text { Cases }\end{array}$ & $\begin{array}{l}\text { influenzae } \\
\text { Sensitive }\end{array}$ & $e^{* *}$ Resistant \\
\hline Ampicillin & 20 & $13(65 \%)$ & $7(35 \%)$ & 7 & $7(100 \%)$ & - \\
\hline Amoxicillin + clavulanic ac & 20 & $18(90 \%)$ & $2(10 \%)$ & 7 & $7(100 \%)$ & - \\
\hline Cefuroxime & 20 & $16(80 \%)$ & $4(20 \%)$ & 7 & $7(100 \%)$ & - \\
\hline Cefotaxime & 20 & $20(100 \%)$ & 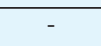 & 7 & $7(100 \%)$ & - \\
\hline$\overline{\text { Meropenem }}$ & 20 & $20(100 \%)$ & - & 7 & $7(100 \%)$ & - \\
\hline Cipro/Levoflox & 20 & $16(80 \%)$ & $4(20 \%)$ & 7 & $7(100 \%)$ & - \\
\hline Cotrimoxazole & 20 & $6(30 \%)$ & $14(70 \%)$ & 7 & $4(57 \%)$ & $3(43 \%)$ \\
\hline Azithromycin & 20 & $12(60 \%)$ & $8(40 \%)$ & 7 & $6(86 \%)$ & $1(14 \%)$ \\
\hline$\overline{\text { Tetracyclines }}$ & 20 & $8(40 \%)$ & $12(60 \%)$ & 7 & $5(71 \%)$ & $2(29 \%)$ \\
\hline$\overline{\text { Rifampicin }}$ & 20 & $17(85 \%)$ & $3(15 \%)$ & 7 & $5(71 \%)$ & $2(29 \%)$ \\
\hline
\end{tabular}

meatitis and haematospermia in 5\%. The Haemophilus spp. group showed greater variability in the reasons for consultation. Of the 5 asymptomatic patients who consulted for screening, in one case we found M. genitalium and in another, $U$. urealyticum.

Duration of symptoms: The duration of the symptoms was less than 1 week for the majority of infections (N. gonorrhoeae, M. genitalium, U. urealyticum and multiple infections). For C. trachomatis, the distribution was more homogeneous. In case of Haemophilus spp. infection, the duration was more than 1 week in the majority of cases, with statistically significant differences compared with the other infections ( $\mathrm{p}=0.002)$.

Type of treatment: The type of treatment was empirical, before cultures or PCR results (46 of 86 cases, 53\%), or based on the antibiogram for the isolated microorganism which usually became available after 4 days (40 of 86 cases, $47 \%$ ).

Clinical cure: Globally we achieved a high percentage of clinical cures, without significant differences between the microorganisms. In 6 cases (2 Haemophilus spp., 2 C. trachomatis, $1 \mathrm{~N}$. gonorrhoeae and $1 \mathrm{U}$. urealyticum) patients went lost at follow up.

Test of cure: PCR was done in all M. genitalium infections, as stated in the guidelines (7), and in all the results were negative. Bacteriological study (Gram stain and culture) was done in $90 \%$ of the cases of Haemophilus spp. infection cases, and in all the results were negative. We also performed a PCR test in all cases of multiple infections, and they were also all negative.

Partner notification and treatment: We carried out the notification and treatment of sexual partners between 75 and $100 \%$ of the cases (7).

\section{Laboratory differences}

Presence of leukocytes: We observed the presence of leukocytes in $75 \%$ of N. gonorrhoeae infections, in $42 \%$ of C. trachomatis infections and in $40 \%$ of multiple infections, but in only $24 \%$ of Haemophilus spp. infections; the differences were not statistically significant. We did not observe leukocytes in any M. genitalium or U. urealyticum infections.

\section{Antibiotic resistance}

Antibiotic resistance was analysed only in cases in which the cultures were positive for $N$. gonorrhoeae or Haemophilus spp. Of the 26 cases positive for N. gonorrhoeae (24 single infections plus 2 multiple infections), we did not observe any case of resistance to ceftriaxone. Of the 27 cases positive for Haemophilus spp. (including those with multiple infections), 20 were positive for $H$. parainfluenzae, of which $70 \%$ were resistant to cotrimoxazole, $60 \%$ resistant to tetracyclines and $40 \%$ resistant to azithromycin. By comparison, among the 7 cases positive for Haemophilus influenzae we found $43 \%$ resistance to cotrimoxazole, $29 \%$ to tetracyclines and rifampicin and $14 \%$ to azithromycin. We observed a $20 \%$ resistance to quinolones in cases of Haemophilus parainfluenzae, without such resistance in Haemophilus influenzae (Table 4).

\section{Multivariate analysis}

Multivariate logistic regression was used to build a predictive model of assignment to the Haemophilus group, 
using a combination of clinical variables. A backward stepwise (conditional) method was used to drop insignificant terms. The final model included as significant predictors the type of unprotected sex being exclusively oral, the main reasons for consultation (testicular pain, no urethral discharge), the duration of symptoms more than one week, and the lack of leukocytes in the urethral sample. The accuracy of the logistic function, measured as the calculated AUC on a ROC curve was 0.834 (0.7170.951 ), and OR of 8.076 (3.391-19.234), showing a sensitivity of $76 \%$ and a specificity of $90 \%$.

\section{Discussion}

To our knowledge this is the first study to compare the characteristics of Haemophilus infections with those of the other sexually transmitted urethral infections. Limitations of this study can be the small sample size and the lack of a representative control group.

Over the past 2 years we have observed an increase in urethral infections due to Haemophilus spp. in our STI department for men, in keeping with other published reviews (8). Motivated by this, we decided to perform a retrospective study to identify the epidemiological, clini$\mathrm{cal}$ and laboratory characteristics of these infections and to compare them with urethral infections by N. gonorrhoeae, C. trachomatis, M. genitalium and U. urealyticum. All male patients who underwent conventional bacteriological and multiplex PCR studies in the urethra at the same time between January 2018 and February 2019 were selected for inclusion in the study, yielding a total of 158 cases. Of these, 86 tested positive for one or more of these microorganisms, including $76(88 \%)$ with infections by a single microorganism and $10(12 \%)$ with multiple infections. This is consistent with the literature, where dual infections have been identified in up to $10 \%$ of men in some studies, expecially among $C$. trachomatis and M. genitalium $(9,10)$. Taking into account that (a) all 27 Haemophilus infections were symptomatic; (b) in 21 of the 27 cases a single microorganism was identified and; (c) all of these patients (except for 2 lost at follow up) had remission of symptoms and negative cultures at control, our first conclusion is that Haemophilus spp. are a sexually transmitted source of symptomatic urethral infection in men. Supporting this, we did not find any cases of Haemophilus infection in the 5 asymptomatic patients who attended for screening.

Füzi was the first to report, in 1980, that Haemophilus could be a cause of sexually transmitted urethritis (11). The 2016 European Guideline on the management of NGU also refers to the fact that Haemophilus spp. are responsible for a small proportion of cases of NGU (7). In our study, Haemophilus spp. were responsible for at least $24.4 \%(21 / 86)$ of the cases and were the second most frequent microorganism to be isolated alone, behind N. gonorrhoeae but ahead of C. trachomatis and M. genitalium. This result does not match previous reports, where C. trachomatis was the most frequent microorganism, followed by N. gonorrhoeae and, thirdly, by M. genitalium (1, 12). In a cross-sectional study conducted by Orellana et al. (13), in which they analyzed 1248 male urethral samples over 3 years, H. parainfluenzae was isolated in 1.76\% and
H. influenzae in $1.12 \%$ of the samples. Probably the frequency of infection by Haemophilus spp. must be related to the sexual habits of the population, and the increasing practice of unprotected oral sex potentially explains these differences in incidence. In our series, $H$. parainfluenzae was more frequent than $H$. influenzae ( $74 \%$ vs $26 \%$ ), which is in concordance with the literature review $(4,8$, 14). Another discrepant finding in comparison to previously published studies, such as Rane's review in 2014, which showed a higher prevalence of Haemophilus urethritis in MSM, is that in our series most infections were in MSW (62\%), with only 33\% in MSM (15). This may be because in our city MSM have access to regular screening and treatment in specific community settings.

In our series, C. trachomatis, M. genitalium and U. urealyticum were less common in MSM than MSW with NGU, which is consistent with the literature (15). All of the Haemophilus group reported unprotected oral insertive sex and $81 \%$ of them denied having had another kind of unprotected sex, which was statistically significantly different compared with the other causes of urethritis. These results were similar to the findings of Deza et al. (2015), who reported that all cases in their series had practiced unprotected insertive oral sex (4). Therefore, it seems clear that the main route of transmission of Haemophilus is via this route (16). These data support the contribution of oropharyngeal exposure to this syndrome. Colonization of the oral cavity by $H$. parainfluenzae and $H$. influenzae in different amounts in healthy individuals is very frequent (17). One aspect that we did not evaluate in men with recurrences, was the need to study partner's oral flora in order to establish a direct relation with oral sex and, also, to determine whether it is necessary to treat sexual partners.

In cases of Haemophilus infection the main reason for consultation varied being the most common dysuria; the difference was statistically significant compared with the other causes of urethritis, for which urethral discharge was the main reason for consultation. In comparison, in the study by Deza et al. the most common clinical presentation among cases of Haemophilus infection was mucopurulent urethral discharge, suggesting potential difficulty in distinguishing causes of urethritis based on symptomatology (4). We did not find any cases of Haemophilus infection in the 5 asymptomatic patients who consulted for screening. Regarding the duration of the symptoms, it was longer than one week in the Haemophilus group but usually less than one week in the other groups, the statistical difference being significant. Leukocytes were frequently present in infections due to N. gonorrhoeae and C. trachomatis as well as multiple infections, but less frequent in infections due to Haemophilus and absent in infections due to $U$. urealyticum and M. genitalium. In this regard it should be noted that there is significant inter- and intra-observer error in counting polymorphonuclear leukocytes, especially in samples with low-grade inflammation (18).

An interesting finding was the multiple resistances to antibiotics in the Haemophilus group, which suggests a need to reconsider which is the best empirical treatment as adjuvant to intramuscular ceftriaxone in patients consulting for urethritis. The most striking finding was that 
$60 \%$ of H. parainfluenzae and $29 \%$ of $H$. influenzae infections were resistant to doxycycline and $40 \%$ of $H$. parainfluenzae and 19\% of H. influenzae infections were resistant to azithromycin, both of which are recommended firstline drugs for the treatment of NGU (19). These percentages are similar to those described in other areas of the world such as East Asia (20). Among the H. parainfluenzae cases there was also a $20 \%$ rate of resistance to quinolones, which exclude them as a therapeutic option. Another drug that may be of special interest in usual practice is amoxicillin in combination with clavulanic acid, since we observed resistance to this combination in only $10 \%$ of $H$. parainfluenzae and none of $H$. influenzae infections. In view of these findings and the high prevalence of resistance either present prior to treatment or developing during treatment, it is important to perform a test of cure to confirm microbiological healing, as recommended in all guidelines for M. genitalium (21). We performed a test of cure in 100\% of cases of M. genitalium and multiple infection, with clinical and microbiological cure confirmed in all cases.

We also performed a test of cure in 90\% (19/21) of the cases of single Haemophilus infection and in all of them we were able to confirm clinical and microbiological cure. An explanation for this high percentage of clinical cures could be that not all the positive cases were treated empirically; in 40 of the 86 cases, we waited for the results, which normally took about 4 days, and then adjusted the antibiotic depending on the antibiogram of the microorganism isolated.

There is good evidence that $U$. urealyticum causes urethritis in some but not all men, the problem being that even the PCR test cannot distinguish between asymptomatic carriers and possible causality (22-24). On the other hand, there are reports of patients with persistent or recurrent Ureaplasma-positive urethritis who have been cured only after their sexual partner received appropriate treatment (23). There is some controversy over the timing of treatment of $U$. urealyticum, especially in asymptomatic patients. In our series, of the 7 positive patients, 6 had symptoms; in addition, all were MSW, with the exception of a single patient with multiple infections who was BSM, and their mean age of 24.5 years was lower than for all other microorganisms. Taking all of this into account, as well as the fact that there is clear evidence that U. urealyticum can affect seminal parameters (25-27), we decided to treat all patients with $U$. urealyticum immediately. To break the chain of transmission, we undertook partner notification and treatment in a large majority of cases, including $75 \%$ of $U$. urealyticum, $100 \%$ of M. genitalium and multiple infection and $95 \%$ of $N$. gonorrhoeae and C. trachomatis.

Multivariate logistic regression model confirmed that patients having mainly oral sex, showing testicular pain but not no urethral discharge neither leukocytes in the urethra, and displaying symptoms for more than one week, were up to eight times more likely to have an Haemophilus as the cause of their urethritis.

Finally, in relation to patients with multiple infections, it is of note that they had the highest mean number of sexual partners, the highest rate of drug use other than alcohol and tobacco and the highest rate of a history of STI.
Main limitation of the study is represented by the retrospective nature of the paper and the absence of a control group; another is the number of patients of our study that, even if it is high for a single Centre in one year, could be implemented to increase statistical significance. Finally, our diagnostic work out was focused on the diagnosis of urethritis not including a screening for concomitant prostatic infections.

\section{Conclusions}

To our knowledge, this is the first study to compare the characteristics of Haemophilus infections with those of the other sexually transmitted urethral infections.

Haemophilus seems to be an aetiological agent of nongonococcal urethritis whose incidence is clearly increasing. Oral insertive sex is the main route of acquisition of Haemophilus urethral infection.

In the Haemophilus group the main reason for consultation varied; this could make diagnosis difficult and the duration of symptoms was clearly longer than for other microorganisms. It is necessary to confirm eradication of infection due to the high rate of antibiotic resistance, for this reason, in the future we should update the guidelines for empirical treatment of urethritis.

Finally, it would be interesting, in cases of recurrence, to detect Haemophilus spp. in the couple's oral cavity and to compare whether the strain coincides with that of the urethra, in order to ascertain whether it is necessary to treat sexual partners.

\section{REFEREnCES}

1. Ito S, Hanaoka N, Shimuta K, et al. Male non-gonococcal urethritis: from microbiological etiologies to demographic and clinical features. Int J Urol. 2016; 23: 325-331.

2. Janier M, Lassau F, Casin I, et al. Male urethritis with and without discharge: a clinical and microbiological study. Sex Transm Dis. 1995; 22:244-52.

3. Bradshaw CS, Tabrizi SN, Read TRH, et al. Etiologies of nongonococcal urethritis: bacteria, viruses, and the association with orogenital exposure. J Infect Dis. 2006; 193: 336-345.

4. Deza G, Martin-Ezquerra G, Gómez J, et al. Isolation of Haemophilus influenzae and Haemophilus parainfluenzae in urethral exudates from men with acute urethritis: a descriptive study of 52 cases. Sex Transm Infect. 2015; 92: 29-31.

5. Hsu MS, Wu MY, Lin TH, et al. Haemophilus parainfluenzae urethritis among homosexual men. J Microbiol Immunol Infect. 2012; 48: 450-452.

6. Clinical and Laboratory Standards Institute (CLSI). Performance Standards for Antimicrobial Susceptibility Testing; Twenty-fifth Informational Supplement. CLSI document M100-S25 (ISBN 156238-990-4). Clinical and Laboratory Standards Institute, 950 West Valley Road, Suite 2500, Wayne, Pennsylvania 19087. USA, 2015.

7. Horner PJ, Blee K, Falk L, et al. European guideline on the management of non-gonococcal urethritis. Int J STD AIDS. 2016; 27:928-37.

8. Vázquez F, Andrés MT, Palacio V. Isolation of Haemophilus influenzae and Haemophilus parainfluenzae in genitourinary infections: a 4year review. Enferm Infec Microbiol Clin. 1996; 14:181-185. 
9. Wetmore CMP, Manhart LEP, Lowens MSP, et al. Demographic, behavioral, and clinical characteristics of men with nongonococcal urethritis differ by etiology: a case comparison study. Sex Transm Dis. 2011; 38:180-6.

10. Gaydos C, Maldeis NE, Hardick A, et al. Mycoplasma genitalium compared to chlamydia, gonorrhoea and trichomonas as an aetiological agent of urethritis in men attending STD clinics. Sex Transm Infect. 2009; 85:438-40.

11. Füzi M. Haemophili in sexually transmitted diseases. Lancet. $1980 ; 2: 476$

12. Manhart LE, Holmes KK, Hughes JP, et al. Mycoplasma genitalium among young adults in the United States: an emerging sexually transmitted infection. Am J Public Health. 2007; 97:1118-25.

13. Orellana MA, Gómez ML, Teresa Sánchez M, FernándezChacón T. Microbiological diagnosis of urethritis in men. 3 years Review. Rev Esp Quimioter. 2009; 22:83.

14. Sturm AW. Haemophilus influenzae and Haemophilus parainfluenzae in nongonococcal urethritis. J Infect Dis. 1986; 153:165-7.

15. Rane VS, Fairley CK, Weerakoon A, et al. Characteristics of acute nongonococcal urethritis in men differ by sexual preference. J Clin Microbiol. 2014; 52:2971-2976.

16. Bradshaw CS, Tabrizi SN, Read TRH, et al. Etiologies of nongonococcal urethritis: bacteria, viruses, and the association with orogenital exposure. J Infect Dis. 2006; 193:336-45.

17. Gonzalez MD, Ledeboer NA Haemophilus. Chapter 38. Manual of Clinical Microbiology, 12th Edition.

18. Smith R, Copas AJ, Prince M, et al. Poor sensitivity and consistency of microscopy in the diagnosis of low grade non-gonococcal urethritis. Sex Transm Infect. 2003; 79:487-90.
19. CDC Sexually Transmitted Diseases Treatment Guidelines. 2021.

20. Deguchi T, Ito S, Hatazaki K, et al. Antimicrobial susceptibility of Haemophilus influenza strains isolated from the urthra of men with acute urethritis and/or epididymitis. J Infect Chemother. 2017; 23:804-7.

21. Horner P, Blee K, O'Mahony C, et al. Clinical Effectiveness Group of the British Association for Sexual Health and HIV. 2015 UK National Guideline on the management of non-gonococcal urethritis. Int J STD AIDS. 2016; 27:85-96.

22. Zhang N, Wang R, Li X, Liu X, Tang Z, Liu Y. Are Ureaplasma spp. a cause of nongonococcal urethritis? A systematic review and meta-analysis. PLoS One. 2014; 9: e113771.

23. Magri V, Boltri M, Cai T, et al. Multidisciplinary approach to prostatitis. Arch Ital Urol Androl. 2019; 90:227-248.

24. Stamatiou K, Magri V, Perletti G, et al. Chronic prostatic infection: Microbiological findings in two Mediterranean populations. Arch Ital Urol Androl. 2019; 91:177-181.

25. Ford DK,Henderson E. Non-gonococcal urethritis due to $T$ mycoplasma (Ureaplasma urealyticum) serotype 2 in a conjugal sexual partnership. Br J Venereal Dis. 1976; 52:341-342.

26. Lee JS, Kim KT, Lee HS, et al. Concordance of Ureaplasma urealyticum and Mycoplasma hominis in infertile couples: impact on semen parameters. Urology. 2013; 81:1219-24.

27. Ma XP, Gao XQ. The effect of Ureaplasma urealyticum on the level of $\mathrm{P} 34 \mathrm{H}$ expression, the activity of hyaluronidase, and DNA fragmentation in human spermatozoa. Am J Reprod Immunol. 2017; 77. doi: 10.1111/aji.12600.

\section{Correspondence}

Alvaro Vives, $M D$

avives@fundacio-puigvert.es

Marco Cosentino, MD (Corresponding Author)

doccosentino@gmail.com

Lluis Bassas, MD

lbassas@fundacio-puigvert.es

Carles Alonso, MD

calonso@fundacio-puigvert.es

Felix Millan

fmillan@fundacio-puigvert.es

Fundacio Puigvert, Barcelona, Spain 